\title{
COVID-19 vaccination and immune thrombocytopenia
}

\author{
A prospective cohort analysis finds a link between the $\mathrm{ChAdOx} 1$ vaccine and an autoimmune disorder known as \\ immune thrombocytopenia-but questions remain and causality is yet to be established.
}

\author{
Allyson M. Pishko, James B. Bussel and Douglas B. Cines
}

W orldwide efforts to control the coronavirus SARS-CoV-2 have led to the most rapid and extensive vaccination program in human history. With all of its benefits, there also comes the potential for side effects, including autoimmune responses. In this issue of Nature Medicine, Simpson and colleagues implicate the AstraZeneca (ChAdOx1) vaccine, but not the Pfizer (BNT162b2) vaccine, in the development of immune thrombocytopenia (ITP) ${ }^{1}$. In interpreting such findings, it is important to consider not only the strengths and limitations of the study but also the wider clinical context and the balance of risks and benefits of vaccination.

Robust, validated data are needed in order to conclude that a vaccine induces a specific disorder. There is now compelling evidence for one such autoimmune disorder, vaccine-induced thrombosis and thrombocytopenia, also known as 'thrombosis thrombocytopenia syndrome'. Approximately 1-3 weeks after initial vaccination with either the ChAdOx1 adenoviral vector vaccine or the Ad26.COV2.S (Johnson \& Johnson) adenoviral vector vaccine, it is estimated that between 1 in 100,000 and 1 in 1,000,000 previously healthy people developed this syndrome, characterized by thrombosis (often involving the cerebral cortical or splanchnic venous systems), thrombocytopenia (often severe), systemic activation of coagulation (elevated concentrations of D-dimer and reduced concentrations of fibrinogen), and the presence of antibodies to platelet factor 4 (ref. ${ }^{2}$ ). The novel and distinctive clinical and serological features of this disorder, combined with preliminary studies implicating the virus in antigen formation $^{3}$, support the proposal of a relationship between vaccination and the autoimmune response.

In other cases, the relationship between a vaccine and a specific disorder has not

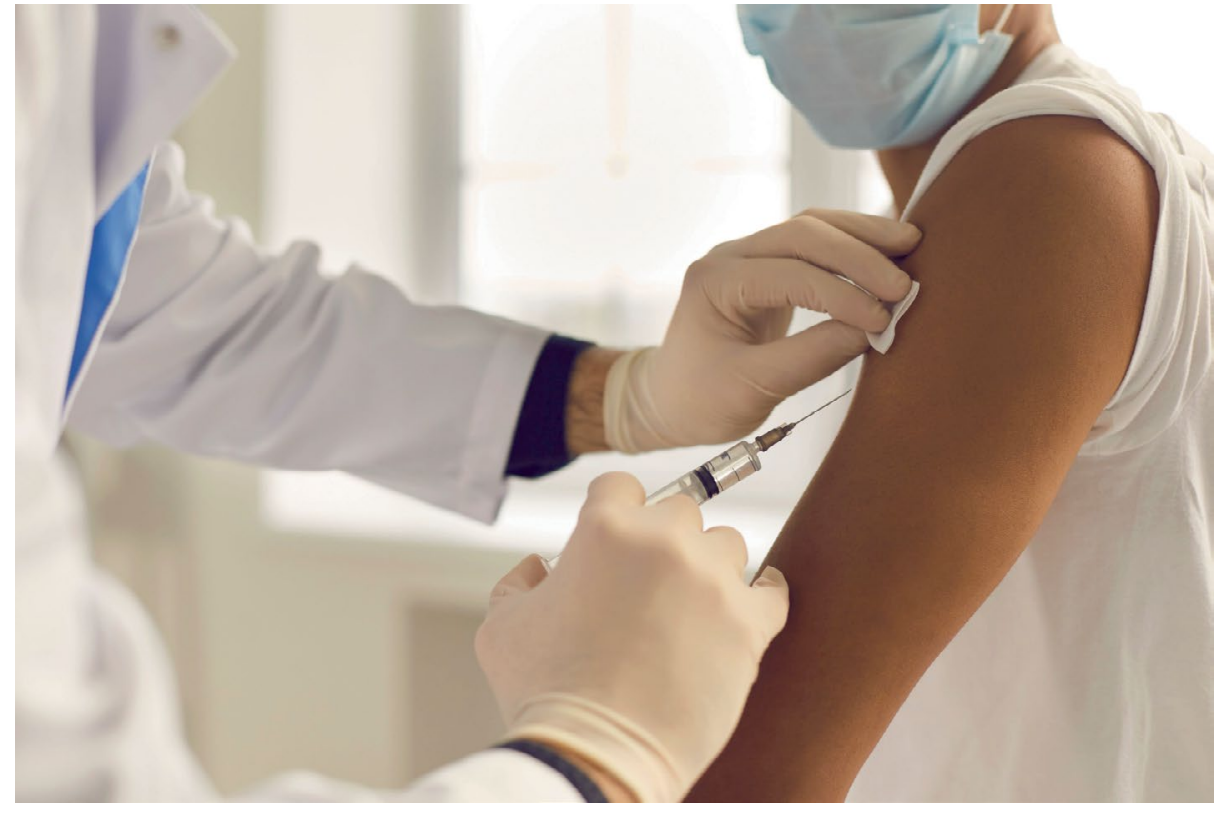

Credit: Roman Lacheev / Alamy Stock Photo

been so clear. Uncertainty is especially likely with an antibody-mediated platelet disorder such as ITP, which is not very rare, may not be serious and is not always reliably diagnosed because of the lack of specific testing. Simpson et al. investigated the connection between ITP and the BNT162b2 and CHAdOxl vaccines using a large national prospective observational cohort (Early Pandemic Evaluation and Enhanced Surveillance of COVID-19 (EAVE II) $)^{4}$. The EAVE II platform seems well suited for this task, as it includes outpatient, inpatient and laboratory data for approximately $99 \%$ of the Scottish population. The investigators' previous work with this database showed a convincing association between first-dose vaccination against COVID-19 and decreased hospital admission for COVID-195.

ITP, however, is a challenging diagnosis, with no unique identifying features when it occurs after vaccination. Unlike vaccine-induced thrombosis and thrombocytopenia, ITP is a diagnosis of exclusion. There is no specific test that confirms the diagnosis, and clinicians therefore rely on the lack of distinguishing features of other diseases, which depends in part on the thoroughness of the evaluation. In some cases, alternative diagnoses may become apparent only during follow-up. For example, a Canadian ITP registry study that incorporated central expert adjudication of diagnosis with long-term follow-up reported that nearly one in seven patients initially diagnosed with ITP were ultimately given an alternative diagnosis ${ }^{6}$; misdiagnoses were also recently reported in children ${ }^{7}$. Perhaps the most reliable inclusive 'diagnostic' test is a robust response to ITP-directed therapies. Therefore, in previous database studies, investigators developed search strategies to 
enhance diagnostic accuracy. These included analysis of diagnostic codes, together with documentation of ITP-directed therapy (such as corticosteroids or intravenous immunoglobulin), in addition to the absence of concurrent and/or subsequent diagnostic codes for other known etiologies of thrombocytopenia (e.g., malignancy, myelodysplastic syndrome or drug-induced thrombocytopenia $)^{8}$. Simpson et al. also attempted to verify the use of ITP-directed therapy but were limited in their access to hospital medication records ${ }^{1}$.

Distinguishing de novo ITP from exacerbation of undiagnosed, pre-existing ITP is another challenge and requires knowledge of pre-vaccination platelet counts, results that were not consistently available in the current study. Three key points are relevant here. First, many patients may have platelet counts as low as 30,000 to 50,000 platelets per microliter while still remaining asymptomatic. Second, transient reductions in platelet counts after infection and vaccination are common, in our experience. Third, pre-vaccination platelet counts are infrequently available for patients who do not otherwise require regular medical attention.

Overall, the concept that vaccination may induce ITP is not new or without precedent. Thrombocytopenia was frequently seen in children exposed to a live attenuated (weakened) vaccine against measles. The average maximum fall in platelet counts approached 100,000 platelets per microliter; the maximum decrease was seen at 1 week after vaccination, and platelet counts generally returned to baseline levels within 3 weeks. Indirect evidence of viral infection of megakaryocytes was seen, suggestive of a component of impaired platelet production'. The attributable risk of ITP, generally mild and resolving within weeks or months, is estimated to be about one case per 40,000 doses of the vaccine against measles-mumps-rubella, which is only slightly higher than that noted after natural infection with the viruses that cause these diseases ${ }^{10}$. In the setting of vaccination against measles-mumps-rubella, a live, albeit weakened, virus is responsible for the 'development' of ITP. To attribute the observations in the current study to cases of de novo ITP, one would have to speculate that both mRNA-based vaccines against SARS-CoV-2 (such as BNT162b2) and adenovirus-based vaccines against SARS-CoV-2 (such as ChAdOx1), or their viral protein cargoes, elicited cross-reactive immunity (e.g., through molecular mimicry), altered a host protein or induced substantial immunological perturbation that led to the emergence of pre-existing autoreactive antibodies. On the one hand, this would seem to be somewhat novel, as ITP did not emerge as a complication of many other 'killed' vaccines given to adults, as assessed through the use of a national registry and case-controlled methodology ${ }^{11}$. On the other hand, the unprecedented number of people vaccinated with these new viral or mRNA vectors in such a short period of time could render previous experience of limited relevance.

Attribution of a rare adverse event to vaccine exposure can exacerbate vaccine hesitancy, with important effects on public health. Seemingly, even exposure of 30,00070,000 recipients to these two vaccines as part of the initial trials was insufficient to identify a risk of ITP, indicative of the need for continued surveillance, as exemplified by the current study, in which the authors estimate 1.13 (95\% confidence interval, 0.62-1.63) additional cases of ITP occur per 100,000 first doses of ChAdOx $1^{1}$. Estimating the 'true effect' is hampered by the multiple challenges associated with the diagnosis of ITP and the low number of incident ITP events observed. Overall, the authors conclude there is no clear evidence of an association of ITP with the first dose of the BNT162b2 vaccine and there is a possible small increased risk of ITP with the ChAdOx1 vaccine ${ }^{1}$.

At the present time, the incidence, severity, duration and responsiveness to treatment of post-vaccine ITP have not been fully established, and further analysis is warranted. Nonetheless, the risk of vaccination-induced ITP at the rate proposed seems to be far lower than the many risks associated with COVID-19 itself. Large international efforts, such as the Brighton Collaboration, are ongoing to continue to assess and improve vaccine safety ${ }^{12}$.

Allyson M. Pishko (D)1,2, James B. Busse ${ }^{3}$ and Douglas B. Cines ${ }^{1,2 \square}$

${ }^{1}$ Department of Medicine, Perelman School of Medicine, University of Pennsylvania, Philadelphia, PA, USA. ${ }^{2}$ Department of Pathology and Laboratory Medicine, Perelman School of Medicine, University of Pennsylvania, Philadelphia, PA, USA. ${ }^{3}$ Department of Pediatrics, Weill Cornell Medicine, New York City, NY, USA.

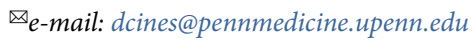

Published online: 9 June 2021

https://doi.org/10.1038/s41591-021-01419-1

References

1. Simpson, C.R. et al. Nat. Med. https://doi.org/10.1038/s41591021-01408-4 (2021).

2. Cines, D.B. \& Bussel, J.B. N. Engl. J. Med. https://doi.org/10.1056/ NEJMe2106315 (2021).

3. Tiede, A. et al. Blood https://doi.org/10.1182/blood.2021011958 (2021).

4. Simpson, C. R. et al. BMJ Open 10, e039097 (2020).

5. Vasileiou, E. et al. Lancet 397, 1646-1657 (2021).

6. Arnold, D. M. et al. Blood Adv. 1, 2414-2420 (2017).

7. Schifferli, A. et al. Blood Adv. 5, 1617-1626 (2021).

8. Moulis, G. et al. Blood 124, 3308-3315 (2014).

9. Oski, F. A. \& Naiman, J. L. N. Engl. J. Med. 275, 352-356 (1966).

10. Mantadakis, E., Farmaki, E. \& Buchanan, G. R. J. Pediatr. 156, 623-628 (2010).

11. Grimaldi-Bensouda, L. et al. Blood 120, 4938-4944 (2012).

12. Condit, R. C. et al. Vaccine 38, 7708-7715 (2020).

Competing interests

A.M.P. has received research funding from an educational grant from Sanofi Genzyme and Novo Nordisk.

J.B.B. has received research support from Amgen; has served as a consultant and/or on advisory boards for Amgen, Dova, Novartis, Rigel, UCB, Momenta-Johnson \& Johnson, Argenix, Principia-Sanofi, RallyBIo and Regeneron; and was a member of the Data Safety Monitoring Board for UCB, CSL Behring. D.B.C. has received research support from Syntimmune/Attenuon and served as a consultant and/or on advisory boards for Novartis, Johnson \& Johnson, Dova, Tavanta and Rigel. 